\title{
A UNIVERSAL DE-NOISING ALGORITHM FOR GROUND-BASED LIDAR SIGNAL
}

\author{
$\mathrm{Xin} \mathrm{Ma}^{\mathrm{a}, \mathrm{b},{ }^{*} \text {, Chengzhi Xiang }}{ }^{\mathrm{a}}$, Wei Gong ${ }^{\text {a }}$ \\ ${ }^{a}$ State Key Laboratory of Information Engineering in Surveying, Mapping and Remote Sensing, Wuhan University, Wuhan 430079, \\ China-maxinwhu@gmail.com, czxiangwhu@gmail.com,gongweiwhu@gmail.com \\ ${ }^{\mathrm{b}}$ Collaborative Innovation Center for Geospatial Technology, Wuhan 430079, China - maxinwhu@gmail.com
}

Commission I, WG I/2

KEY WORDS: Ground-based lidar, Universal de-noising, Signal segmentation, Dual field-of-view Mie lidar

\begin{abstract}
:
Ground-based lidar, working as an effective remote sensing tool, plays an irreplaceable role in the study of atmosphere, since it has the ability to provide the atmospheric vertical profile. However, the appearance of noise in a lidar signal is unavoidable, which leads to difficulties and complexities when searching for more information. Every de-noising method has its own characteristic but with a certain limitation, since the lidar signal will vary with the atmosphere changes. In this paper, a universal de-noising algorithm is proposed to enhance the SNR of a ground-based lidar signal, which is based on signal segmentation and reconstruction. The signal segmentation serving as the keystone of the algorithm, segments the lidar signal into three different parts, which are processed by different de-noising method according to their own characteristics. The signal reconstruction is a relatively simple procedure that is to splice the signal sections end to end. Finally, a series of simulation signal tests and real dual field-of-view lidar signal shows the feasibility of the universal de-noising algorithm.
\end{abstract}

\section{INTRODUCTION}

Ground-based lidar is an effective remote sensing tool in atmosphere remote sensing(Kovalev and Eichinger, 2004). The raw lidar signal can directly show the information of the atmospheric condition. Yet, the noise in signal is inevitable, leading to difficulties in digging more information about aerosol, cloud and other interesting factors. The signal-to-noise ratio (SNR) can sharply decreases due to the existence of noise at high altitude.

To reduce the noise level, many methods have been adopted. The low-pass filters are mostly accepted de-noising methods. The multiple-pulse accumulation and averaging can reduce the noise level to $1 / \sqrt{n}$, yet the temporal and spatial resolution are greatly limited(Milton et al., 1992). The other low-pass filters, including Fourier transform (FT), empirical mode decomposition (EMD), and wavelet transforms (WTs), are widely used in signal de-noising(Rabiner and Gold, 1975, Huang et al., 1998, Flandrin et al., 2004, Mallat and Hwang, 1992). However, each individual method has its own feature that can suit a unique situation. The FT cannot work well when dealing with lidar signal, the EMD results in errors when encounter with lidar signal that has huge signal fluctuation(Tian et al., 2014), while the WTs have to select a proper wavelet base to adapt different signals(Fang et al., 2005). Currently, there is no universal de-noising method that can fit all lidar signals. In this paper, we introduced a universal de-noising method for ground-based lidar signal based on signal segmentation and reconstruction. Considering the variation of lidar signal caused by weather and other influences, we segment the signal into different parts, which can be processed by different de-noising method based on their own characteristics. Then, the simulation signal and real lidar signal experiments are introduced and discussed to show the feasibility of the proposed method.

\section{METHOD DESCRIPTION}

EMD is an adaptive de-nosing method, but has some innate drawbacks, such as overshoot and edge effect. The de-noising accuracy of WT is high, but it has to select a proper wavelet base varied with signal. The proposed de-noising method combined the advantages of EMD and WT, but has its own characteristic, which can be described as follows:

a. Background noise deduction. The background noise of lidar signal includes the background light noise and detector noise. The former one can be defined as the averaged signal intensity at the farthest altitude of the each received signal. The latter one can be measured as a profile when covering the telescope to make sure that no light arriving at the detector. Then, the pretreated signal can be obtained by removing the background noise.

b. Layer detection. The layer signal has large signal fluctuation, which is unfavorable to the procession of EMD. Thus, if the layer is determined before signal de-noising and processed by a proper method, the de-noising accuracy will be greatly improved. And the algorithms for layer detection is quite mature, which will not be discussed here(Pal et al., 1992).

c. Signal segmentation. Considering the fact that the signal SNR at low altitude is quite high, we define the noise-free signal that is reserved without de-noising processing. Firstly, the lidar equation should be simplified as a linear one as follows:

$$
\ln [P(r)]=a \times \ln (r)+b
$$

where $\ln [\mathrm{P}(\mathrm{r})]=$ the natural logarithm of $\mathrm{P}(\mathrm{r})$

$\ln (r)=$ the natural logarithm of $r$

$$
a, b=\text { coefficients of the least square linear fitting }
$$

The signal variation $\mathrm{V}\left(\mathrm{r}_{i}\right)$ is defined as follows:

\footnotetext{
* Corresponding author. Xin Ma, maxinwhu@gmail.com
} 


$$
\mathrm{V}\left(\mathrm{r}_{i}\right)=\sum_{\mathrm{j}=\mathrm{i}-\mathrm{n}}^{\mathrm{j}=\mathrm{i}+\mathrm{n}} \frac{\mathrm{abs}\left\{\ln \left[\mathrm{P}\left(\mathrm{r}_{j}\right)\right]-\mathrm{a}_{i} \ln \left(\mathrm{r}_{j}\right)-\mathrm{b}_{i}\right\}}{\ln \left[\mathrm{P}\left(\mathrm{r}_{j}\right)\right]}
$$

where $\quad i=$ the center bin number of a piece of the local signal The calculation number of the variation in the signal per piece is $2 \mathrm{n}+1$. The linear fitting coefficients will differ for every piece of local signal.

A pre-set threshold $\sigma$ is given based on the experiment results. If the $\mathrm{V}\left(\mathrm{r}_{m}\right)$ is smaller than $\sigma$, the noise-free signal is defined as the bins that lower than $r_{m-n}$.

Then the signal is segmented into three parts: the noise-free one, the layer one and the remaining one.

d. Signal processing. Each signal piece should be processed by a proper de-nosing method, according to its own feature. The noise-free signal should not be processed. The layer signal is de-noised by discrete wavelet transform (DWT) with Symlet 4 as its wavelet base and a hard thresholding to guarantee the preservation of the sharp feature of layer signal. At last, the remaining signal is handled by the SG-EMD(Boudraa and Cexus, 2007), which is a combination of SG filter and EMD, obtaining a better de-noising effect.

e. Signal splicing. At last, the several signal segments can be spliced into an integrated noise-reduced signal

\section{DATA ILLUSTRATION}

To validate the effectiveness of the proposed de-noising method, the simulation signal and real lidar signal are both adopted in this article.

\subsection{Simulation signal}

The simulation signal can evaluate the de-noising effect quantitatively. And the simulated lidar signal $P_{s}(r)$ can be expressed as follows:

$P_{s}(r)=P_{t}(r)+e(r)$

where $\quad P_{t}(r)=$ the true and pure signal without noise $\mathrm{e}(\mathrm{r})=$ the added Gaussian white noise

Figure 1 shows the simulated noisy signal, which has two layers at the altitude of $2200 \mathrm{~m}$ and $4300 \mathrm{~m}$.

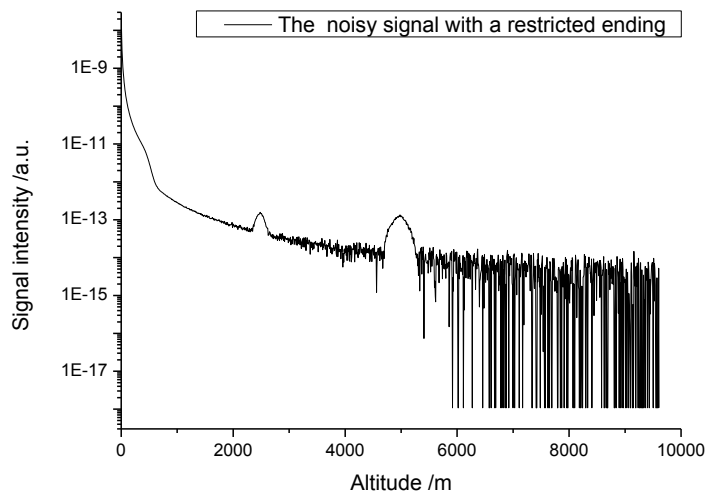

Figure 1. The simulated noisy signal

\subsection{Dual field-of -view Mie lidar signal}

The dual field-of-view Mie lidar (DFML) provides the possibility of evaluation for real signal. The original purpose of this DFML is to increase the dynamic range of a lidar signal using two independent receiving channels: one with large FOV aimed at a lower altitude, named as near-range signal, while the other one with small FOV aimed at high altitude, named as farrange signal. Actually, a large FOV can bring in more background light noise. In contrast, a small FOV will limit the noise level at a limited altitude. Considering the valid altitude for a far-range signal, the altitude of the signal adopted is limited from 2500 to $5000 \mathrm{~m}$. Figure 2 shows the local nearrange and far-range raw signal at the same altitude after the signal normalization..

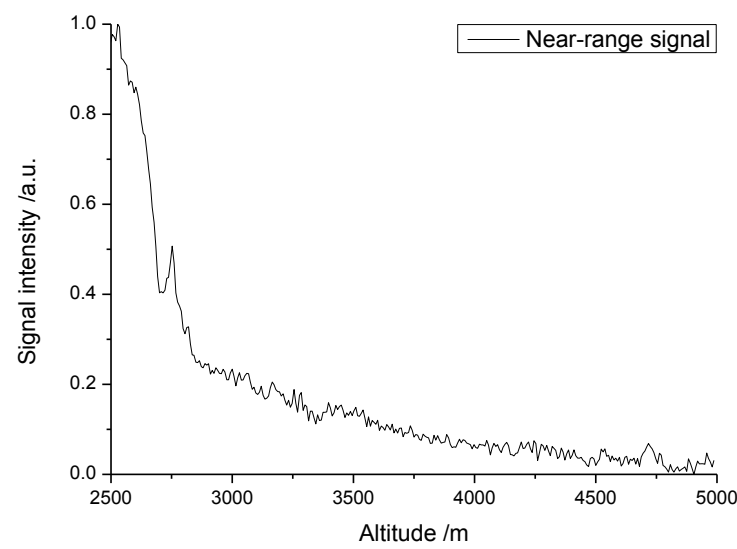

Figure 2(a). The near-range signal

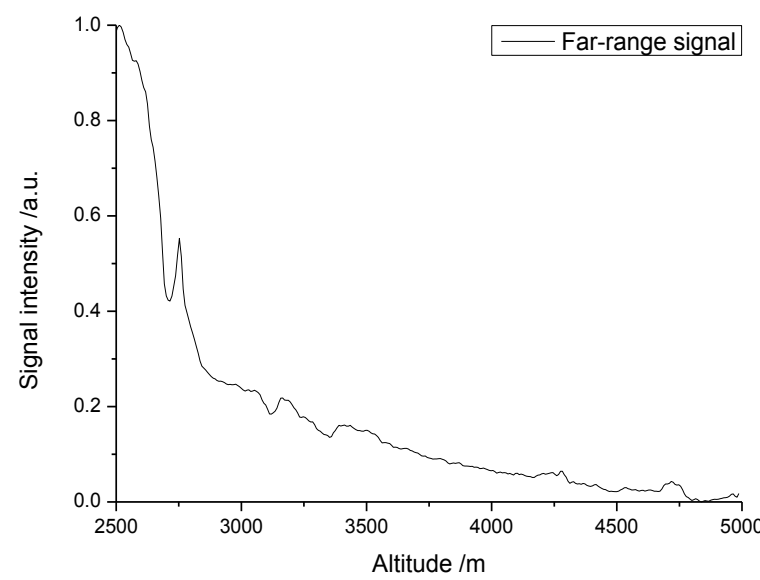

Figure 2(b). The far-range signal at the corresponding altitude

The far-range and near-range signals show the same tendency and even show a high similarity in some local areas. Yet, the former is obviously clean with high SNR, which is the basis for the evaluation of the noise reduction effect of the different denoising methods for DFML signals.

\section{RESULTS AND DISCUSSION}

\subsection{Simulation signal de-noising}

The simulated signal in figure 1 is processed by different denosing methods, including EMD, DWT and the proposed method. Figure 3 shows the de-noising result with the proposed method, it can clearly seen that the noise level is greatly limited compared with figure 1 . 


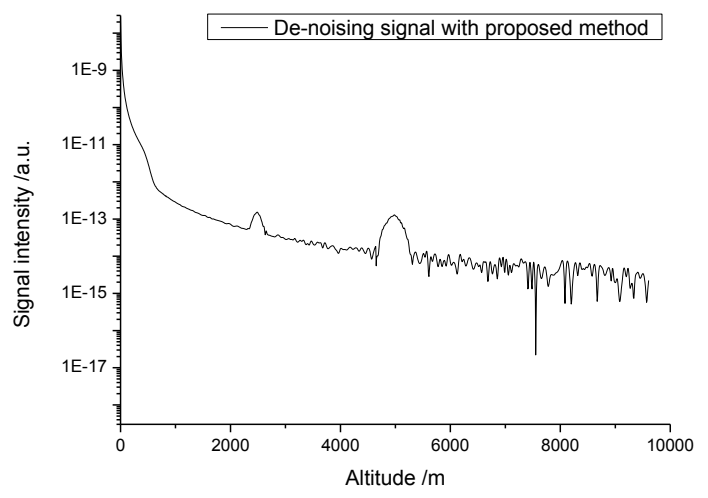

Figure 3. The de-noised simulation signal by the proposed method

Then, the SNR (Signal-to-Noise Ratio) is defined to evaluate the de-noising effect as follows:

$$
S N R=10 \times \lg \frac{\sum_{i=1}^{n} P_{d e}^{2}\left(r_{i}\right)}{\sum_{i=1}^{n}\left(P_{s}\left(r_{i}\right)-P_{t}\left(r_{i}\right)\right)^{2}}
$$

where $\quad P_{d e}\left(r_{i}\right)=$ the de-noised signal at altitude $\mathrm{r}_{\mathrm{i}}$

$$
n=\text { the signal length }
$$

The results in table 1 show that EMD cannot work well as the others, since the simulation signal has obvious layer that will cause serious overshoot effect. DWT with $\mathrm{db} 2$ as the wavelet base is relatively satisfied but still can reach the best de-noising result. The main reason is that the $\mathrm{db} 2$ has a big difference with the lidar signal with layers. The results demonstrated that a proper base for DWT is much important when dealing with various type of lidar signal.

\begin{tabular}{|l|l|l|}
\hline & $\begin{array}{l}\text { Single } \\
\text { layer }\end{array}$ & $\begin{array}{l}\text { Multiple } \\
\text { layer }\end{array}$ \\
\hline Noise-added signal & 15.51 & 34.78 \\
EMD & 20.11 & 39.22 \\
DWT & 24.54 & 42.52 \\
The proposed method & 27.32 & 49.27 \\
\hline
\end{tabular}

Table 1. The signal SNR after processed by different methods

\subsection{DFML signal de-noising}

The presence of DFML provides the possibility of an evaluation in actual experiments. The far-range signal with a small FOV with little noise in a limited range can be used as a noise-free signal, and the near-range signal with a large FOV can be considered as a polluted signal. Figure $2 \mathrm{a}$ and $2 \mathrm{~b}$ shows that the near- and far-range signals display similar trends but with markedly different noise levels, and the former has a higher SNR. The similarity of the signals lies in the fact that the two telescopes are aimed at almost the same atmosphere, whereas the different noise level is caused by the different FOVs.

Thus, the de-noised near-range signal can be compared with the far-range signal, and the effect of the proposed de-noising algorithm and the other methods can also be tested at the limited range. The pseudo SNR serves as an important indicator, which can be defined as follows:

$$
S N R=10 \times \lg \frac{\sum_{i=1}^{n} P_{f a r}^{2}\left(\mathrm{r}_{i}\right)}{\sum_{i=1}^{n}\left[P_{d e}\left(\mathrm{r}_{i}\right)-P_{f a r}\left(\mathrm{r}_{i}\right)\right]^{2}}
$$

where $\quad P_{f a r}\left(r_{i}\right)=$ the far-range signal at altitude $\mathrm{r}_{\mathrm{i}}$

A group of typical normalized DFML signals is shown in figure 2. The de-noising result of the near-range signal shown in figure 2(a) processed by the proposed method is shown in figure 4 , where the noise-level of the de-noising signal is greatly reduced compared with the near-range signal. The de-noised signal retains the local detail features when referred to the far-range signal shown in figure 2(b).

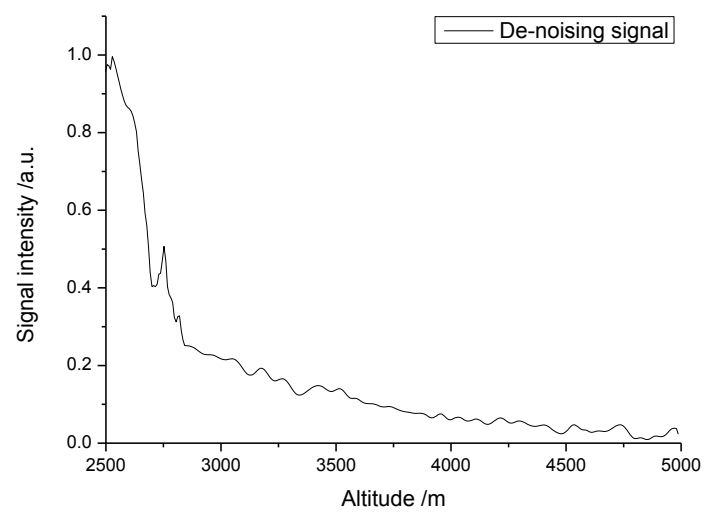

Figure 4. De-noised signal using the proposed method

Table 2 lists the SNR for the different types of de-noising methods. Generally, the DWT works more steadily than the EMD mainly because the chosen local signals share a steady trend. The DWT with a proper wavelet function can certainly work well when dealing with this type of signal. With regard to the local signal, the overshoot effect may be reduced when no large fluctuation occurs. Thus, the de-noising effect of the EMD is comparable with those of the other methods. The combination of the simulated and experimental signals indicates that a normal EMD cannot deal with signals collected in a cloudy situation unless improved by some means. With regard to the proposed method, the combination of the advantages of the EMD and DWT making the de-noising effect enhance. Similar to the result of the simulated signal, the proposed method still occupies a superior status, yielding the highest SNR.

\begin{tabular}{|l|l|l|}
\hline & 1 & 2 \\
\hline Near-range signal & 40.76 & 38.64 \\
EMD & 48.22 & 44.05 \\
DWT & 48.03 & 47.02 \\
The proposed method & 49.59 & 49.36 \\
\hline
\end{tabular}

Table 2. The pseudo SNR after processed by different methods

\section{CONCLUSTIONS}

Because of the variation in the lidar signal, no universal denoising method can fix all situations. In this article, a de-noising algorithm has been proposed based on signal segmentation and reconstruction. The simulation and real experiments showed the validation of the proposed method. Further work should be focused on the efficiency of the de-noising algorithm. 


\section{ACKNOWLEDGEMENTS}

This work was supported by National Nature Science Foundation of China(NFFC) (41127901); the Fundamental Research Funds for the Central Universities (Grant No. 2014619020201) and Program for innovative Research Team in University of Ministry of Education of China (IRT1278)

\section{REFERENCES}

Boudraa A. O., Cexus J. C., 2007. EMD-based signal filtering. IEEE T. Instrum. Meas. 56: 2196-2202.

Fang H. T., Huang D. S., Wu Y. H., 2005. Antinoise approximation of the lidar signal with wavelet neural networks[J]. Appl. Optics, 1077-1083.

Flandrin P., Rilling G., Goncalves P., 2004. Empirical mode decomposition as a filter bank. IEEE Signal Proc. Let. 11: 112114 .

Huang N. E., Shen Z., Long S. R., et al. 1998. The empirical mode decomposition and the Hilbert spectrum for nonlinear and non-stationary time series analysis. Proceedings of the Royal Society of London. Series A: Mathematical, Physical and Engineering Sciences, 454: 903-995.

Kovalev V. A., Eichinger W. E., 2004. Elastic lidar: theory, practice, and analysis methods. John Wiley \& Sons.

Mallat S., Hwang W. L., 1992. Singularity detection and processing with wavelets. IEEE T. Inform. Theory. 38: 617-643.

Milton M., Woods P., Jolliffe B., et al. 1992. Measurements of toluene and other aromatic hydrocarbons by differentialabsorption LIDAR in the near-ultraviolet. Appl. Phys. B-lasers O. 55: 41-45.

Pal S. R., Steinbrecht W., Carswell A. I., 1992. Automated method for lidar determination of cloud-base height and vertical extent[J]. Appl. Optics. 31: 1488-1494.

Rabiner L. R., Gold B. 1975. Theory and application of digital signal processing. Englewood Cliffs, NJ, Prentice-Hall, Inc., 1975. 777 p., 1.

Tian P. F., Cao X. J., Liang J. N., et al. 2014. Improved empirical mode decomposition based denoising method for lidar signals[J]. Opt. Commun. 325: 54-59. 